4. Wehrl M, Michels W. A method for testing the cleaning of MIS robotic instruments. Central Service 2013;3:202-207.

5. Wehrl M, Albers G, Bühler K, et al. Round robin tests conducted by the working group Da Vinci (AG DaVinci) to establish a method for testing the cleaning of MIS robotic instruments. Central Service 2014;3:173-179.

6. Carter A, Krüger S, Schmidt V, et al. Guideline compiled by the DGKH, DGSV and AKI for validation and routine monitoring of automated cleaning and disinfection processes for heat-resistant medical devices as well as advice on selecting washer-disinfectors. Central Service 2007;15:1-48.

7. Tollefson MK, Frank I, Gettman MT. Robotic-assisted radical prostatectomy decreases the incidence and morbidity of surgical site infections. Urology 2011;78:827-831.

8. Southworth PM. Infections and exposures: reported incidents associated with unsuccessful decontamination of reusable surgical instruments. J Hosp Infect 2014;88:127-131.

9. MAUDE-Manufacturer and user facility device experience. US Food and Drug Administration website: http://www.accessdata. fda.gov/scripts/cdrh/cfdocs/cfMAUDE/detail.cfm? mdrfoi_id=5878988\&pc=NAY. Published 2016. Accessed April 10,2017

10. Wendorf KA, Kay M, Baliga C, et al. Endoscopic retrograde cholangiopancreatography-associated AmpC Escherichia coli outbreak. Infect Control Hosp Epidemiol 2015;36:634-642.

\section{The Puzzle of Volume, Coverage, and Application Time in Hand Disinfection}

To the Editor-Pires et $\mathrm{al}^{1}$ provide some interesting data and suggest that using $3 \mathrm{~mL}$ hand rub and rubbing the volume into both hands for either 15 or 30 seconds yields a similar bacterial reduction on healthcare worker (HCW) hands. Although they did not look at the coverage of both hands after 15 or 30 seconds (eg, with a fluorescent dye), the data nevertheless suggest that once the hand rub is fairly distributed to both hands within 15 seconds, further rubbing does not add to the overall efficacy of $60 \%$ isopropanol.

The general application of $3 \mathrm{~mL}$, however, deserves further consideration. Based on data from France, the use of $3 \mathrm{~mL}$ is regarded by $99.8 \%$ of HCW as sufficient for complete hand coverage. ${ }^{2}$ At the same time, all studies indicate that on regular hands the application of $3 \mathrm{~mL}$ keeps hands moist for more than 30 seconds, ${ }^{3,4}$ sometimes even for 60 seconds. ${ }^{2}$ The other side of this correlation is that an HCW will require a volume between 1.7 and $2.1 \mathrm{~mL}$ depending on the type of hand $\mathrm{rub}^{3}$ if hands are to remain moist for 30 seconds. If the setting used by Pires et al with $3 \mathrm{~mL}$ per application for a 15 -second duration were transferred into clinical practice, hands would still be moist after 15 seconds and would need to dry during the next 15-45 seconds before further patient care activities. What would an HCW be able to do during the drying time? Also, having alcohol-moist hands can result in burns because static electricity may cause ignition of the vapor from the hand rub, although this is extremely uncommon.

The goal certainly remains to make hand hygiene easier for augmented compliance especially in hospital units with many indications per healthcare worker and per shift. ${ }^{5}$ But how can this goal be achieved? If hands are rubbed until dry and shorter application times are desired, smaller volumes per application will be needed on average size hands, (eg, 1.5 or $2 \mathrm{~mL}$ ). A volume of $1.5 \mathrm{~mL}$ is considered sufficient for hand coverage by $95.8 \%$ of HCWs, and a volume of $2 \mathrm{~mL}$ is considered sufficient by $98.5 \%{ }^{2}$ A volume of $\sim 2 \mathrm{~mL}$ would also be acceptable to users. ${ }^{6}$ Averagesized hands are dry after $\sim 30$ seconds. But based on efficacy data obtained with European Standard EN 1500, these volumes usually fail the EN 1500 efficacy requirement with mean $\log _{10}$ reductions between 3.05 and 4.03. ${ }^{3,4}$

Healthcare workers will certainly welcome shorter but equally effective hand disinfection. Recommending a smaller volume, however, should be assured from various viewpoints. This new volume should ensure coverage of both hands; this technique should be easy to perform and be effective on small and large hands. Coverage of hands can quite easily be measured with a fluorescent dye. At the same time, the simplicity of the rub-in technique can be evaluated. These measurements could provide the basis for testing the efficacy of such a change (eg, according to EN 1500).

It may be time to review some parameters of current efficacy testing standards. Hand size currently has no place in EN 1500. Why not have 3 subgroups of subjects with small, medium, and large hands, respectively? A proposal for hand-size classification has been made already. ${ }^{7}$ Each participant would initially have to determine how much volume is necessary to keep both hands wet (eg, for 20 or 30 seconds), resulting in a specific test volume per subject and application time. This volume would later be used for efficacy testing against the reference procedure. A second parameter for review may be the type of contamination in EN 1500. Having half of the hands in an Escherichia coli broth is associated with a high organic load on both hands. If the broth contained a black dye, hands would probably be classified as "visibly soiled" and should be washed instead of treated with a hand rub. ${ }^{8}$ A different type of contamination with a high inoculum but a substantially lower amount of organic load may better resemble clinical practice'; it may even show that $2 \mathrm{~mL}$ of a hand rub is very effective.

\section{ACKNOWLEDGMENTS}

Financial support: No financial support was received in relation to this article. Potential conflict of interest: The author has worked in the past for Bode Chemie GmbH, Hamburg, Germany.

\section{Günter Kampf, MD}

Affiliation: University Medicine Greifswald, Institute for Hygiene and Environmental Medicine, Greifswald, Germany. 
Address correspondence to Günter Kampf, MD, University Medicine Greifswald, Institute for Hygiene and Environmental Medicine, WalterRathenau-Straße 49 A, 17475 Greifswald, Germany (guenter.kampf@unigreifswald.de).

Infect Control Hosp Epidemiol 2017;38:880-881

(C) 2017 by The Society for Healthcare Epidemiology of America. All rights reserved. 0899-823X/2017/3807-0021. DOI: 10.1017/ice.2017.85

\section{REFERENCES}

1. Pires D, Soule H, Bellissimo-Rodrigues F, Gayet-Ageron A, Pittet D. Hand hygiene with alcohol-based hand rub: how long is long enough? Infect Control Hosp Epidemiol 2017:1-6.

2. Girard R, Aupee M, Erb M, Bettinger A, Jouve A. Hand rub dose needed for a single disinfection varies according to product: a bias in benchmarking using indirect hand hygiene indicator. J Epidemiol Global Health 2012;2:193-198.

3. Macinga DR, Shumaker DJ, Werner HP, et al. The relative influences of product volume, delivery format and alcohol concentration on dry-time and efficacy of alcohol-based hand rubs. BMC Infect Dis 2014;14:511.

4. Kampf G, Marschall S, Eggerstedt S, Ostermeyer C. Efficacy of ethanol-based hand foams using clinically relevant amounts: a cross-over controlled study among healthy volunteers. BMC Infect Dis 2010;10:78.

5. Azim S, Juergens C, McLaws ML. An average hand hygiene day for nurses and physicians: The burden is not equal. Am J Infect Control 2016;44:777-781.

6. Wilkinson MAC, Ormandy K, Bradley CR, Fraise AP, Hines J. Dose considerations for alcohol-based hand rubs. J Hosp Infect 2017;95:175-182.

7. Bellissimo-Rodrigues F, Soule H, Gayet-Ageron A, Martin Y, Pittet D. Should alcohol-based handrub use be customized to healthcare workers' hand size? Infect Control Hosp Epidemiol 2016;37:219-221.

8. World Health Organization. WHO Guidelines on Hand Hygiene in Health Care. First Global Patient Safety Challenge Clean Care is Safer Care. Geneva: WHO; 2009.

9. Macinga DR, Beausoleil CM, Campbell E, et al. Quest for a realistic in vivo test method for antimicrobial hand-rub agents: introduction of a low-volume hand contamination procedure. Appl Environ Microbiol 2011;77:8588-8594.

\section{Understanding the $\beta$-Lactam/Inhibitor of $\beta$-Lactamase Combinations: Reassessment for Better Antimicrobial Stewardship}

To the Editor-The $\beta$-lactamases are plasmid-encoded or chromosomally encoded enzymes that hydrolyze $\beta$-lactam antibiotics. Those that are plasmid-mediated can be rapidly transferred between bacterial genera and can put in check the successful use of $\beta$-lactam agents. The $\beta$-lactam/inhibitor of $\beta$-lactamase (BL/IBL) combinations are a class of agents with proven success in treating infections caused by bacteria producing $\beta$-lactamases, mostly the conventional-spectrum enzymes. ${ }^{1}$
The prevalence of gram-negative bacteria resistant to broad-spectrum $\beta$-lactams has increased alarmingly in past decades, including those extended-spectrum $\beta$-lactamase (ESBL)producing organisms with poorer clinical outcomes than more susceptible organisms. ${ }^{2}$

Unequivocally, carbapenems have a relatively high clinical success rate among patients infected with ESBL-producing organisms. ${ }^{3}$ However, indiscriminate carbapenem use has contributed to the increased emergence of carbapenem-resistant Enterobacteriaceae (CRE). ${ }^{4}$

Because it is crucially important to conserve the usefulness of carbapenems in the era of antimicrobial resistance, a survey was conducted to monitor the contemporary crude prevalence of resistance rates for BL/IBL combinations against Escherichia coli, Klebsiella, and Proteus species displaying a conventional or ESBLenzyme spectrum, including those presenting a carbapenemresistance profile but not a carbapenemase production relation.

Enterobacterial isolates were recovered from inpatients between January 1 and December 26, 2016, at a tertiary hospital in Porto Alegre, Southern Brazil. Escherichia coli, Klebsiella, and Proteus species were selected because other minor prevalent enterobacterial species such as Enterobacter, Providencia, Serratia, and Citrobacter freundii have an intrinsic resistance to amoxicillin/ clavulanate. Biochemical tests using a MicroScan automated system (Beckman Coulter, Brea, CA) were used to identify E. coli, Klebsiella, and Proteus species and to determine their resistance rates to amoxicillin/clavulanate (AMC), ampicillin/sulbactam (SAM), and piperacillin/tazobactam (TZP). All selected enterobacterial isolates were confirmed for the presence of an ESBL enzyme using a synergistic test applying clavulanic acid, as previously described. ${ }^{2}$ Isolates with reduced susceptibility to any carbapenem agent were tested using a synergistic test applying phenyl-boronic acid and ethylenediaminetetraacetic acid to detect Klebsiella pneumoniae carbapenemase (KPC) and metallo- $\beta$-lactamase enzyme, in that order. Only CRE isolates with a negative result for any carbapenemase were included in this study.

A total of 942 isolates were included in this survey; 878 isolates (93.2\%) had a community profile: 441 E. coli $(50.2 \%)$; 213 Proteus mirabilis (24.3\%); 210 K. pneumoniae (23.9\%); and $14 \mathrm{~K}$. oxytoca (1.6\%). In addition, 62 isolates $(6.6 \%)$ had an ESBL-producing spectrum: $53 \mathrm{~K}$. pneumoniae (85.5\%), 8 E. coli (12.9\%); and 1 P. mirabilis (1.6\%). Only 2 isolates (0.2\%), K. pneumoniae, and E. coli, had a carbapenem-resistance profile. Of these isolates, $591(62.7 \%)$ were recovered from urine, $174(18.5 \%)$ were recovered from blood, $92(9.8 \%)$ were recovered from respiratory secretions, 19 (2\%) were recovered from catheter tip, and $66(7 \%)$ were recovered from elsewhere.

Resistance rates to AMC, SAM, and TZP for each categorized group (community-based, ESBL-producing, or CRE profile) are shown in Table 1. Overall, among the BL/IBL combinations, TZP was the most active combination (14.6\% of resistance rate), followed by AMC (32.3\% of resistance rate) and SAM (51.9\% of resistance rate). The greatest potency of activity was shown by TZP 